\title{
DO DROWSY DRIVER DRUGS DIFFER?
}

\author{
Timothy Brown ${ }^{1}$, Andrew Spurgin ${ }^{12}$, Gary Milavetz ${ }^{12}$, Gary Gaffney ${ }^{13}$, Robin Johnson ${ }^{4}$ \\ ${ }^{1}$ National Advanced Driving Simulator, University of Iowa, Iowa City, IA \\ ${ }^{2}$ College of Pharmacy, University of Iowa, Iowa City, IA \\ ${ }^{3}$ Carver College of Medicine, University of Iowa, Iowa City, IA \\ ${ }^{4}$ Advanced Brain Monitoring, Carlsbad, CA \\ timothy-1-brown@uiowa.edu
}

\begin{abstract}
Summary: This research paper explores how different drug mechanisms within a single class of drugs can produces different profiles of driving impairment. Prior research has failed to consider these mechanistic differences and often utilizes less controlled study methodologies. The potential impact of differing mechanistic effects is important for practitioners but remains unclear for most drugs. Twentynine licensed drivers in good general health completed one of two miniSim ${ }^{\mathrm{TM}}$ studies using a validated, standardized, driving impairment scenario. Both drugs caused degradation in lateral control measures of standard deviation of lane position (SDLP) and number of lane departures, however only diphenhydramine was found to cause a significant change in steering bandwidth. The studied drugs differed in their effects on all longitudinal driving measures with diphenhydramine effecting speed and alprazolam effecting the standard deviation of speed. Difference in therapeutic mechanism of action results in differing pharmacodynamic driving performance outcomes. This analysis reinforces the importance of careful consideration of a drug's specific mechanism of action when considering a sedating drug's impact on a patient's ability to safely operate a motor vehicle.
\end{abstract}

\section{OBJECTIVES}

With almost half of the population of the United States using at least one prescription drug in the past month, and over a fifth using at least three in the past month, there is a corresponding rise in the number of people driving while under the influence of these medications (National Center for Health Statistics., 2014). Many of these drugs may not affect driving performance, however some will. There is limited information regarding the pharmacodynamic effects of drugs on driving performance, no regulatory requirements to investigate the effects. Since one of the main focuses of treatment is to get the patient to a point where they can resume many of their activities of daily living (ADLs), it is imperative that practitioners have access to solid evidence-based science regarding when a patient may be safe to operate a motor vehicle after consuming a potentially impairing substance.

The current body of literature is limited mostly to on-road studies conducted in Europe. While these studies are useful, they also have limitations, many of which can be addressed by using an advanced research-driving simulator. One of the major limitations is the lack of control over the driving environment. Having a sensitive, and repeatable, driving environment is essential to getting meaningful findings out of driving studies. On-road studies are unable to ensure exact reproduction between trials (ensuring same temperature, weather, state of the road, state of the vehicle, etc.). Another limitation is the safety of subjects, staff, and the public when studying 
potentially impaired drivers. Simulation using advanced research driving simulators offers a high face-validity, reproducible, and safe driving environment to test impairment.

There is currently no standardized or required method to test the potential impairing effects of drugs that are active in the central nervous system. These drugs may cause functional changes in the brain relative to executive functioning, autonomic functioning (i.e., operational control), decision-making, and a number of other skills that are required to safely drive a car. Typically, physicians and pharmacists are required to counsel patients on medications that may cause the side effects of drowsiness, dizziness or altered mental acuity. This consists of instructing the patient that the drug may impair driving performance, and that they should not operate heavy machinery until they know how they drug is going to affect them individually.

While drugs may have very different mechanisms of action, the observed impairing effects on driving tend to be similar by drug class. Diphenhydramine crosses the blood brain barrier and exerts CNS depressant activity causing predictable drowsiness (Gengo et.al., 1988), and the accompanying desire to sleep. This effect has been shown to cause significant changes in common driving impairment measures - standard deviation of lane position, SD speed, etc. (Weiler et al , 2001).

Alprazolam is a short-acting benzodiazepine-class medication that exerts its pharmacologic activity by modulating the GABA receptor. The benzodiazepine class of drugs is mainly used to control generalized anxiety disorder (though they also have an indication for sleeplessness). In treating anxiety, the drug produces a CNS depression that can cause the side effects of drowsiness or dizziness. Benzodiazepines have been shown to be associated with an increased risk in road-traffic accidents (Barbone et al. 1998), though their effect on individual driving performance measures is less clear.

\section{METHODS}

Data sources for this analysis come from two different studies completed at the National Advanced Driving Simulator (NADS), at the University of Iowa. 20 (12 male, 8 female, mean age $25.2 \mathrm{yo}$ ) subjects participated in a study looking at the effects of alprazolam (Xanax ${ }^{\circledR}$ ) on driving performance. Subjects were randomized to receive either $1 \mathrm{mg}$ alprazolam or a placebo (both of which were overencapsulated in identical opaque capsules) in a double-blind placebo controlled study design. A separate group of participants $(n=9,3$ male, 6 female, mean age 33.6yo) were enrolled and administered 50mg diphenhydramine ( 2 tablets of $25 \mathrm{mg}$ Benadryl ${ }^{\circ}$, Johnson \& Johnson).

At peak drug effect, subjects in both studies completed a $45 \mathrm{~min}$ drive through a validated, standardized scenario using a NADS miniSim ${ }^{\mathrm{TM}}$ research driving simulator. The driving scenario has been validated at detecting impairment caused by the consumption of alcohol (Kay et al., 2013). The scenario incorporates a number of driving speeds and environments including an urban environment, a residential area, a high-speed interstate section, and a rural two-lane highway all at night. For this analysis, data was used from six driving events with similar driving demand: urban driving, suburban driving, interstate curves, rural driving, and rural driving on a long curve tangent. 


\section{Dependent Measures}

Dependent measures in this study were grouped into those related to lateral and those related to longitudinal control. Lateral control measures included average lane position relative to the center of the lane, standard deviation of lane position (SDLP), bandwidth of the frequency content in the steering signal, and number of lane departures per minute. A natural log transformation was performed for SDLP. Longitudinal control measures included speed relative to the speed limit, standard deviation of speed, percent of time driver was more than $5 \mathrm{mph}$ greater than the speed limit, and percent of time driver was more than $5 \mathrm{mph}$ less than the speed limit. A Logit transformation was made for both percent measures.

\section{Participants}

Forty-six adult participants in good general health who possessed a valid driver's license and drive at least 5000 miles per year enrolled and completed all study procedures. Participants compensated for completion all study procedures.

\section{Apparatus}

Driving simulation was carried out in the National Advanced Driving Simulator (NADS) miniSim $^{\text {TM }}$ Research Driving Simulator (see Figure 1), a PC-based and validated research driving simulator (Kay et al., 2013).

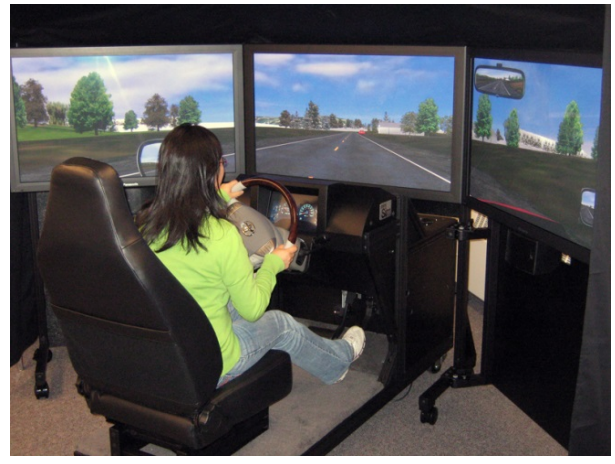

Figure 1. Three-channel quarter cab miniSim

\section{Experimental Procedures}

Participants were recruited for this study using the NADS subject registry and through emails to the university community. Individuals who expressed interest were screened over the phone including a description of the study and a determination of eligibility. Upon arrival, the study was described and participants provided signed informed consent, provided consent of video release, and completed a payment voucher for compensation. Subjects then drove a familiarization drive to get them acclimated to the driving simulator and also screened for simulator sickness. A wellness survey was administered to all participants to assess common symptoms of simulator sickness. If no simulator sickness was detected their remaining dosing 
visits were scheduled. Prior to administration of study drug a urine drug screen, and pregnancy test if female, were administered. Once these screening mechanism were completed, the subject was ready to start the testing phase of the protocol. In the diphenhydramine arm of the study participants drove a baseline study drive prior to drug administration. After completion of the baseline drive, they were administered the study drug and waited an hour and fifteen minutes until the drug was at peak effect. After this waiting period, the participant was escorted back to the driving simulator to complete the study drive under the influence of diphenhydramine. In the alprazolam study however, the study drug was administered immediately after morning screening, and allowed two hours to take effect. Since this portion of the study was placebocontrolled, the participant only completed one study drive per visit. Once the study drive was complete, all subjects completed a wellness survey to ensure they did not have any simulator sickness. After completing all procedures, all subjects driven home by either study staff or a friend or relative.

\section{Scenarios}

The 35-40 minute driving scenario consisted of three approximate 10-minute segments of urban, interstate and rural driving followed by a ten-minute straight rural driving environment. The data collection drives started with an urban segment composed of a two-lane roadway through a city with posted speed limits of 25 to 45 with signal-controlled and uncontrolled intersections. An interstate segment followed that consisted of a four-lane divided expressway with a posted speed limit of $70 \mathrm{mph}$. Following a period in which drivers followed the vehicle ahead, they encountered infrequent lane changes associated with the need to pass several slower-moving trucks. The drives concluded with a rural segment that was composed of a two-lane undivided road with curves, followed by a gravel road segment, and then a 10-minute section of straight rural driving. The scenario was designed to provide representative driving environments where impaired driving crashes are over represented in the FARS data, but without crash imminent events. Three equivalent versions of the scenario were created to minimize learning effects.

\section{RESULTS}

The SAS General Linear Models (GLM) procedure was used to perform an Analysis of Variance (ANOVA) on the parametric dependent measures. Post-hoc tests were used where appropriate for significant main effects and utilized the post-hoc tukey-test. Although event was included in the model, only drug effects will be reported. Results are grouped by lateral and longitudinal control variables. Cohen's d effect sizes were calculated for each measure.

For lateral control measures, as can be seen in Table 1, there were significant effects for diphenhydramine for SDLP, steering bandwidth and number of lane departures. The table also shows that for Alprazolam there are significant effects for SDLP and number of lane departures. For both drugs, there was no difference in average lane position. For SDLP and number of lane departures the effect size was larger for alprazolam, but for steering bandwidth, there was a small effect for Alprazolam but a medium effect for diphenhydramine. A comparison of the means for each condition where there was at least one drug effect are provided in Table 2. 
Table 1. Drug effects on lateral control

\begin{tabular}{|l|c|c|c|c|}
\cline { 2 - 5 } \multicolumn{1}{c|}{} & \multicolumn{2}{c|}{ Diphenhydramine } & \multicolumn{2}{c|}{ Alprazolam } \\
\cline { 2 - 5 } \multicolumn{1}{c|}{} & $\mathrm{p}$ & Cohen's d & $\mathrm{p}$ & Cohen's d \\
\hline Average Lane Position & 0.4544 & 0.08 & 0.3163 & 0.06 \\
\hline Standard Deviation of Lane Position & 0.0300 & 0.47 & 0.0003 & 0.57 \\
\hline Steering Bandwidth & 0.0289 & 0.42 & 0.3216 & 0.16 \\
\hline Number of Lane Departures & 0.0081 & 0.38 & 0.0041 & 0.60 \\
\hline
\end{tabular}

Table 2. Comparison of mean lateral control performance by drug

\begin{tabular}{|l|c|c|c|c|}
\cline { 2 - 5 } \multicolumn{1}{c|}{} & \multicolumn{2}{c|}{ Diphenhydramine } & \multicolumn{2}{c|}{ Alprazolam } \\
\cline { 2 - 5 } \multicolumn{1}{c|}{} & Active & Baseline & Active & Baseline \\
\hline Ln Standard Deviation of Lane Position & 3.59 & 3.43 & 3.60 & 3.41 \\
\hline Raw Standard Deviation of Lane Position $(\mathrm{cm})$ & 36.4 & 31.0 & 36.7 & 30.2 \\
\hline Steering Bandwidth $(\mathrm{Hz})$ & 1.89 & 1.74 & 1.62 & 1.56 \\
\hline Number of Lane Departures & 1.23 & 0.80 & 1.48 & 0.66 \\
\hline
\end{tabular}

For longitudinal control measures, as can be seen in Table 3 Table 1, there were significant effects for diphenhydramine for average speed and for percent speed high. For both effects, the Cohen's $\mathrm{d}$ effect size indicates a medium sized effect. The table also shows that for alprazolam there are significant effects for standard deviation of speed, but that the effect was small. For both drugs, there was no difference in percent speed low. A comparison of the means for each condition where there was at least one drug effect are provided in Table 4.

Table 3. Drug effects on longitudinal control

\begin{tabular}{|l|c|c|c|c|}
\cline { 2 - 5 } \multicolumn{1}{c|}{} & \multicolumn{2}{c|}{ Diphenhydramine } & \multicolumn{2}{c|}{ Alprazolam } \\
\cline { 2 - 5 } \multicolumn{1}{c|}{} & $\mathrm{p}$ & Cohen's d & $\mathrm{p}$ & Cohen's d \\
\hline Average Speed relative to the Speed Limit & 0.0200 & 0.51 & 0.1313 & 0.30 \\
\hline Standard Deviation of Speed & 0.5228 & 0.14 & 0.0392 & 0.19 \\
\hline Percent Speed High & 0.0176 & 0.43 & 0.1593 & 0.18 \\
\hline Percent Speed Low & 0.2732 & 0.20 & 0.6774 & 0.07 \\
\hline
\end{tabular}

Table 4. Comparison of mean longitudinal control performance by drug

\begin{tabular}{|l|c|c|c|c|}
\cline { 2 - 5 } \multicolumn{1}{c|}{} & \multicolumn{2}{c|}{ Diphenhydramine } & \multicolumn{2}{c|}{ Alprazolam } \\
\cline { 2 - 5 } \multicolumn{1}{c|}{} & Active & Baseline & Active & Baseline \\
\hline Average Speed relative to the Speed Limit (m/s) & 1.6 & 0.6 & 1.5 & 0.5 \\
\hline Standard Deviation of Speed (m/s) & 1.8 & 1.7 & 2.0 & 1.8 \\
\hline Logit Percent Speed High & -0.88 & -2.82 & -2.33 & -3.20 \\
\hline Raw Percent Speed High & $40 \%$ & $26 \%$ & $33 \%$ & $25 \%$ \\
\hline
\end{tabular}




\section{CONCLUSIONS}

Having demonstrated difference in effects between these two CNS depressant drugs, it is useful to consider how the different mechanisms of action relate to observed differences. Diphenhydramine produced increases in SDLP, lane departures, steering bandwidth, average speed, and percentage of time more than $5 \mathrm{mph}$ over the speed limit; whereas alprazolam produced increases in SDLP, lane departures, and variability in speed. As can be seen both drugs resulted in a degradation in lane keeping performance, but only diphenhydramine changed the frequency of steering inputs (greater bandwidth). Although both drugs affected speed control, diphenhydramine resulted in drivers traveling faster; whereas, alprazolam only increased the variability in speed indicating less precise speed maintenance. The mechanisms of these two drugs may offer some explanation.

The drowsiness induced by diphenhydramine is most often described as a generalized desire to sleep. Individuals on this drug are often very aware of this effect and may be compensating by an increased frequency of steering input. This combined with their lack of success at maintaining lane position may indicate steering centered on drifts from one side of the lane to the other. The greater speed chosen by drivers under the influence of diphenhydramine may also reflect a compensatory choice by them to get to the end of the drive sooner, before they fall asleep. In contrast, the drowsiness induced by alprazolam is most often described as a general relaxation. Individuals on this drug may not be cognizant of the impairing effects while on the drug by nature of its therapeutic action (anxiolytic). In this state, these individuals would exhibit less compensatory action that results in less precise control consistent with the changes observed.

The fact that two CNS depressant drugs that cause "drowsiness" produce differing impairment profiles for driving performance illustrates the importance of a clear understanding of the underlying mechanisms associated with the drug's impairing effects. Understanding these differences will help clinicians provide evidence based counseling to their patients. Further research is needed to better understand the underlying mechanisms of impairment within and across drug classes.

\section{ACKNOWLEDGMENTS}

The National Institutes of Drug Abuse funded this research under contract numbers: HHSN271201100009C and HHSN27120100019C. These findings represent the views of the authors. The authors would like for further recognize Chris Schwarz, NADS, and Chris Berka for their contribution to this research.

\section{REFERENCES}

Barbone, F., Mcmahon, A., Davey, P., Morris, A., Reid, I., Mcdevitt, D., \& Macdonald, T. (1998). Association of road-traffic accidents with benzodiazepine use. The Lancet,13311336. 
Gengo, F., Gabos, C., \& Miller, J. (n.d.). The pharmacodynamics of diphenhydramine-induced drowsiness and changes in mental performance. Clinical Pharmacology and Therapeutics, $15-21$

National Center for Health Statistics (2014). Health, United States, 2013: With Special Feature on Prescription Drugs. Hyattsville, MD. 2014.

Kay GG, Ahmad O, Brown T and Veit A.(2013). Comparison of the miniSim and STISim driving simulators for the detection of impairment: An alcohol validation study. (2013) Driving Assessment, 2013: Proceedings of the Seventh International Driving Symposium on Human Factors in Driver Assessment, Training, and Vehicle Design 192-197

Weiler JM, Bloomfield JR, Woodworth GG, Grant AR, Layton TA, Brown TL, et al.(2000) Effects of Fexofenadine, Diphenhydramine, and Alcohol on Driving Performance: A Randomized, Placebo-Controlled Trial in the Iowa Driving Simulator. Ann Intern Med. .Annals of Internal Medicine, 132, 354-363. 